\title{
What's in complementary medicines?
}

\section{Geraldine Moses \\ Adjunct associate professor, School of Pharmacy, University of Queensland, Brisbane \\ Consultant clinical pharmacist, Mater Health Services, Brisbane \\ Consultant pharmacist, Australian Dental \\ Association}

\section{Keywords}

complementary medicine, drug labelling, drug regulation, excipients

Aust Prescr 2019;42:82-3 https://doi.org/10.18773/ austprescr.2019.024
Many millions of dollars are spent on complementary medicines by Australians every year, but these medicines are not without their risks. Adverse reactions are well documented such as the hepatotoxicity of black cohosh and comfrey. There are also potential interactions between complementary medicines and prescription drugs, such as those that occur with St John's wort ${ }^{2}$ and the catechins in green tea. ${ }^{3}$

Consumers and health professionals are increasingly aware that drug interactions with complementary medicines can alter the efficacy of conventional medicines and increase their toxicity. This is of particular concern during cancer chemotherapy.

Predicting drug interactions is complex and requires a comprehensive knowledge of the pharmacodynamics and pharmacokinetics of the ingredients of complementary medicines and the drugs they may interact with. Insight into the nature of drug-interaction literature and the strengths and weaknesses of drug-interaction checkers is also required. It is also helpful to understand the wide range of ingredients present in complementary medicines that contribute to drug interactions such as citrus bioflavonoids and black pepper. To do all this and assess drug interactions accurately, one needs to know all the ingredients in the products involved, not just the active ingredient.

Finding out the ingredients in a medicine should be as easy as reading the label or product information. This certainly is the case with registered medicines in Australia as they receive considerable scrutiny from the Therapeutic Goods Administration (TGA) after clinical trials. Their constituents must be declared, and the product information and associated labelling approved before the drug can be marketed.

In contrast, complementary medicines undergo little of this scrutiny. They are mostly classified by the TGA as 'listed' medicines, which by definition may only contain unscheduled ingredients with few public health concerns. ${ }^{4}$ The regulatory pathway for listed medicines does not require proof of contents or efficacy or pre-approval of labelling and product information before marketing. A listing request is simply submitted online to the TGA and, once the necessary fee is paid, marketing is approved. As a result, the stated ingredients of complementary medicines are accepted on trust. There is no guarantee that the ingredients list is accurate.
Few Australian consumers or health professionals are familiar with how our drug regulatory system works. Most are unaware that complementary medicine labels may not be entirely trustworthy or that 'natural health products', especially those sourced from overseas, may contain undeclared adulterants such as banned substances or prescription drugs. In addition, most health professionals have little structured education regarding complementary medicines or of pharmacognosy (the science of the derivation of drugs from plants or other natural sources). They may not appreciate which details may be incorrect, misleading or neglected in the labelling and packaging of complementary medicines, or understand the potential therapeutic or toxicological effects of the constituents.

Despite these shortcomings, the labelling can still provide helpful and valid information for documenting complementary medicines within a patient's medication history. As with conventional medicines, it is insufficient to note only the active ingredients (e.g. 'Patient takes ginkgo, turmeric and selenium'). Instead, the brand name of the complementary medicine should be recorded, to show exactly which product the patient is taking along with its dose and duration. Without knowing the product name, a full assessment of which substances the patient is exposed to and their drug-interaction potential cannot be made.

In Australia listed and registered products are issued with an AUST L or AUST R number when included in the Australian Register of Therapeutic Goods (ARTG). This number must be printed on the front face of their packaging to indicate the product's legitimate inclusion in the ARTG. The number can be used to search the ARTG, via the TGA's website, to access the manufacturer's public declaration of ingredients. Absence of an AUST L or R number from the packaging may indicate a lack of permission for marketing the product in Australia.

The label should give the country of origin. Products from overseas are subject to manufacturing and regulatory processes that may be different from those of Australia so the level of assurance about the quality of the products will vary.

The more outlandish the therapeutic claims, the more likely the product may be unsafe or unreliable. The label can provide useful details about the formulation and any co-formulated ingredients such as alcohol 
or caffeine. It may reveal which herbs are used in a herbal medicine.

For homeopathic products the label may give information about the potency. What is the base? If it is alcohol, what is the concentration? What are the active substances, and do they pose risks of cross allergy or toxicity? The 2015 NHMRC Statement on Homeopathy provides health professionals with a useful summary of this type of product. ${ }^{5}$

Although the ingredient lists on complementary medicines may be viewed with suspicion, it is often necessary to accept them at face value when making decisions with patients. In addition to assessing the potential risks and benefits of the main ingredient, attention should be paid to the co-formulated ingredients as these substances, often ignored, can represent a significant source of adverse effects and drug interactions.

A contemporary example is black pepper (Piper nigram, $P$. longum) containing piperine which is often included in turmeric products to enhance curcuminoid bioavailability. Piperine is a moderate inhibitor of cytochrome P450 (CYP) 3A4 and 2D6 and can therefore interact with a wide range of conventional medicines. ${ }^{6}$ Other compounds like this are found in schisandra fruit and goldenseal root which also inhibit CYP3A4 and 2D6.7 Catechins in green tea inhibit several organic anion-transporting polypeptides (drug transporters) and the antioxidant resveratrol inhibits CYP2C9 and CYP2D6. ${ }^{8}$ Caffeine is another commonly ignored ingredient because people are often unaware that it is present in Camellia sinensis, guarana and yerbe mate. Yet the quantity of caffeine contained in a complementary medicine may cause significant stimulant effects.

Complementary medicines these days increasingly contain patentable preparations of so-called 'proprietary blends' of phytochemicals. ${ }^{9}$ Even though the individual substances were originally derived from a natural source, these commercial combinations do not occur in nature and have never been administered to humans to determine safety, and so the pharmacological repercussions of their medicinal use are unknown. ${ }^{9}$ We rely on consumers and health professionals to play an active role in monitoring the safety of these complementary medicines. Adverse events should be reported to the TGA.

In summary, although we cannot know for sure what is contained in most complementary medicines, we can know the products being taken. Product names and formulations should be documented in the patient's medication history rather than just the active ingredients so a comprehensive assessment can be made of the adverse effect and druginteraction potential. $<$

Conflict of interest: none declared

\section{REFERENCES}

1. Stournaras E, Tziomalos K. Herbal medicine-related hepatotoxicity. World J Hepatol 2015;7:2189-93. https://doi.org/10.4254/wjh.v7.i19.2189

2. Borrelli F, Izzo AA. Herb-drug interactions with St John's wort (Hypericum perforatum): an update on clinical observations. AAPS J 2009;11:710-27. https://doi.org/10.1208/ s12248-009-9146-8

3. Roth M, Timmermann BN, Hagenbuch B. Interactions of green tea catechins with organic anion-transporting polypeptides. Drug Metab Dispos 2011;39:920-6. https://doi.org/10.1124/dmd.110.036640

4. Therapeutic Goods Administration. Overview of listed complementary medicines [Internet]. 2017 Oct 29. www.tga.gov.au/overview-listed-complementary-medicines [cited 2019 May 1]

5. National Health and Medical Research Council. NHMRC statement: statement on homeopathy [Internet]. March 2015. www.nhmrc.gov.au/about-us/publications/evidenceeffectiveness-homeopathy-treating-health-conditions [cited 2019 May 1]
6. Lee SH, Kim HY, Back SY, Han HK. Piperine-mediated drug interactions and formulation strategy for piperine: recent advances and future perspectives. Expert Opin Drug Metab Toxicol 2018;14:43-57. https://doi.org/10.1080/17425255.2018.1418854

7. Sprouse AA, van Breemen RB. Pharmacokinetic interactions between drugs and botanical dietary supplements. Drug Metab Dispos 2016;44:162-71. https://doi.org/10.1124/ dmd.115.066902

8. Detampel P, Beck M, Krähenbühl S, Huwyler J. Drug interaction potential of resveratrol. Drug Metab Rev 2012;44:253-65. https://doi.org/10.3109/03602532.2012.700715

9. Gurley BJ, Yates CR, Markowitz JS. “....Not intended to diagnose, treat, cure or prevent any disease." 25 years of botanical dietary supplement research and the lessons learned. Clin Pharmacol Ther 2018;104:470-83. https://doi.org/10.1002/cpt.1131

\section{FURTHER READING}

Memorial Sloan Ketting Cancer Center. About herbs database [Internet]. www.mskcc.org/cancer-care/diagnosis-treatment/ symptom-management/integrative-medicine/herbs/search [cited 2019 May 1] 